\title{
Special feature: computational statistics and machine learning
}

\author{
Hiroshi Yadohisa ${ }^{1} \cdot$ Wataru Sakamoto ${ }^{2}$
}

Published online: 23 April 2019

(c) Japanese Federation of Statistical Science Associations 2019

The Japanese Society of Computational Statistics (JSCS) published the Englishlanguage Journal of the Japanese Society of Computational Statistics (JJSCS) for 30 years. During that time the journal played the important role of promoting and extending the use of computational statistics for data science in Japan. JJSCS and the Journal of the Japan Statistical Society (JJSS) were succeeded by our new journal, the Japanese Journal of Statistics and Data Science (JJSD). JSCS continues to be responsible for its publication as a member of the Japanese Federation of Statistical Science Association.

In commemoration of the founding of JJSD, JSCS is publishing our second special feature, which focuses on relationships and collaborations between computational statistics and machine learning. In the area of machine learning, numerous innovative methods have been proposed for statistical computing, pattern recognition, image processing, and other purposes. In particular, deep learning has been remarkably attractive as a technique for artificial intelligence. Most such methodologies have been developed in combination with statistical approaches, including probability distribution theories, multivariate analysis, Bayesian statistics, and some optimization techniques.

This special feature comprises three contributions of scientists working actively in the area.

Sudo et al. (2019) are concerned with reciprocal recommendation, which has attracted great attention. They propose a new method of reciprocal recommendation by using a graph-embedding technique called cross-domain matching correlation (CDMCA) (Shimodaira 2015). They show numerically that CDMCA with a similarity-based weighting scheme provides a high-quality reciprocal recommendation.

Hiroshi Yadohisa

hyadohis@mail.doshisha.ac.jp

Wataru Sakamoto

w-sakamoto@okayama-u.ac.jp

1 Doshisha University, Kyoto, Japan

2 Okayama University, Okayama, Japan 
Ishioka et al. (2019) propose a method to detect a hotspot cluster by using spatial statistics. They use a zero-suppressed binary detection diagram (Minato 1993) to detect a hotspot. To evaluate the proposed method, they compare it with the flexscan and echelon methods.

Bunji and Okada (2019) propose a new class of item response models called linear ballistic accumulation (LBA) item response theory (IRT). LBA is one of the models of response times (Brown and Heathcote 2008). Bunji and Okada show numerically that the proposed model can obtain the corresponding parameter estimates compared with the diffusion IRT model (Ratcliff 1978).

We are grateful to all reviewers for their help in the process of refereeing the contributions and for sharing their time and knowledge. We also want to thank again all authors who have contributed interesting original work to this special feature.

\section{References}

Brown, S. D., \& Heathcote, A. (2008). The simplest complete model of choice response time: Linear ballistic accumulation. Cognitive Psychology, 57(3), 153-178.

Bunji, K. \& Okada, K. (2019). Item response and response time model for personality assessment via linear ballistic accumulation. Japanese Journal of Statistics and Data Science. https://doi.org/10.1007/ s42081-019-00040-4

Ishioka, F., Kawahara, J., Mizuta, M., Minato, S., \& Kurihara, K. (2019). Evaluation of hotspot cluster detection using spatial scan statistic based on exact counting. Japanese Journal of Statistics and Data Science. https://doi.org/10.1007/s42081-018-0030-6.

Minato, S. (1993). Zero-suppressed BDDs for set manipulation in combinatorial problems. In Proceedings of the 30th ACM/IEEE Design Automation Conference (pp. 272-277).

Ratcliff, R. (1978). A theory of memory retrieval. Psychological Review, 85(2), 59-108.

Shimodaira, H. (2015). A simple coding for cross-domain matching with dimension reduction via spectral graph embedding. arXiv:1412.8380.

Sudo, K., Osugi, N., \& Kanamori, T. (2019). Numerical study of reciprocal recommendation with domain matching. Japanese Journal of Statistics and Data Science. https://doi.org/10.1007/s4208 1-019-00033-3.

Publisher's Note Springer Nature remains neutral with regard to jurisdictional claims in published maps and institutional affiliations. 